\title{
Coupler induced monopole component and its minimization in deflecting cavities
}

\author{
P. K. Ambattu, ${ }^{1,2}$ G. Burt, ${ }^{1,2}$ A. Grudiev, ${ }^{3}$ V. Dolgashev, ${ }^{4}$ and A. Dexter ${ }^{1,2}$ \\ ${ }^{1}$ Cockcroft Institute, WA4 4AD, United Kingdom \\ ${ }^{2}$ Lancaster University, LA1 4YR, United Kingdom \\ ${ }^{3}$ CERN, Geneva CH-1211, Switzerland \\ ${ }^{4}$ SLAC, Menlo Park, California 94025, USA \\ (Received 24 December 2012; published 4 June 2013)
}

\begin{abstract}
Deflecting cavities are used in particle accelerators for the manipulation of charged particles by deflecting or crabbing (rotating) them. For short deflectors, the effect of the power coupler on the deflecting field can become significant. The particular power coupler type can introduce multipole rf field components and coupler-specific wakefields. Coupler types that would normally be considered like standard on-cell coupler, waveguide coupler, or mode-launcher coupler could have one or two rf feeds. The major advantage of a dual-feed coupler is the absence of monopole and quadrupole rf field components in the deflecting structure. However, a dual-feed coupler is mechanically more complex than a typical single-feed coupler and needs a splitter. For most applications, deflecting structures are placed in regions where there is small space hence reducing the size of the structure is very desirable. This paper investigates the multipole field components of the deflecting mode in single-feed couplers and ways to overcome the effect of the monopole component on the beam. Significant advances in performance have been demonstrated. Additionally, a novel coupler design is introduced which has no monopole field component to the deflecting mode and is more compact than the conventional dual-feed coupler.
\end{abstract}

DOI: 10.1103/PhysRevSTAB.16.062001

PACS numbers: 29.27.-a, 84.40.-x, 41.85.Ct

\section{INTRODUCTION}

Disk loaded waveguides were originally applied to the acceleration of charged particle bunches using radio frequency (rf) power. Later the fundamental deflecting mode of a disk loaded waveguide was considered and used for bunch deflection and rotation. A ground breaking application was particle separation utilizing the "LOLA" structures developed at SLAC [1]. In 1988, Palmer proposed the use of deflecting modes for bunch rotation allowing luminosity recovery in colliders with crossing angles [2]. In 2007 the concept was demonstrated at the KEK B factory [3]. Crab cavities are currently being proposed for several new machines including ILC, CLIC, and LHC [4-6]. More recent applications of rf deflectors and crab cavities include bunch length and phase-space diagnostics [7], production of ultrashort $\mathrm{x}$ rays [8], and beam position monitoring [9].

The differing field distributions for the operating modes of deflecting and accelerating cavities naturally lead to differences in the way the cavities are developed and optimized. For a $\mathrm{TM}_{01}$-like accelerating mode the magnetic field is concentrated near the equator with zero azimuthal variation, while the electric field is centralized on axis between disk apertures, fringing between adjacent irises

Published by the American Physical Society under the terms of the Creative Commons Attribution 3.0 License. Further distribution of this work must maintain attribution to the author(s) and the published article's title, journal citation, and DOI. and concentrated at the iris surfaces. As a result of this, surface electric $(E)$ and magnetic $(B)$ fields are maximized at differing locations and an on-axis beam will interact only with the electric field. On the other hand, for a deflecting mode, both $E$ and $B$ fields are concentrated between the disk apertures, although there peaks appear at mutually orthogonal azimuthal points on the iris. The surface fields are both maximized on the cavity irises and the beam will interact with transverse components of both fields.

Radio frequency coupling ports transfer the energy between the cavity structure and the waveguide connected to external devices such as the rf source or a load. The fundamental mode couplers are critical components in high power $\mathrm{rf}$ structures such as the CLIC linac prototypes and breakdown test structures $[10,11]$ where the peak power can be as high as $\sim 60 \mathrm{MW}$. At this power level, low surface magnetic field cavity designs are ideal as they minimize the rf pulsed heating. For deflecting dipole cavities, the peak surface magnetic field is found on the iris. This allows high power rf coupling slots to be put on the cell equator without increasing the peak magnetic field, which can be an important issue when coupling to the accelerating mode. For such coupling slots on deflecting cavities, the magnetic field strength in the slot stays below the magnetic field on the iris. At peak powers below $30 \mathrm{MW}$ where most deflecting cavities operate, the major concerns are multipole field components, the trapped higher order modes in the coupler, and the coupler size. Waveguide or mode-launcher couplers are used very commonly [12,13] and might either have single or dual feed [14]. Standard couplers connect the feed waveguide 
directly to the equator of the end cell through a slot, waveguide couplers couple to the structure through an end cell supporting a TE mode, and mode-launcher couplers transform the rectangular waveguide into an enlarged beampipe or circular waveguide which is then coupled to the structure through a matching cell [13]. Even though the standard and waveguide couplers both use rectangular waveguides, due to the plane of coupling they are physically different. Discarding the practical components like flanges, bellows, wakefield dampers, etc., for a given number of cells, a standard coupler is the most compact in longitudinal plane while the waveguide coupler is the most compact in the transverse plane. The mode-launcher coupler is the least compact longitudinally; however, it has the advantage that the same coupler can be used for a broad range of structures. Symmetric dual-feed operation prevents excitation of monopole and quadrupole field components of the deflecting mode by the coupler providing there is a good phase match between the feeds. For a single-feed coupler, although the monopole and quadrupole components of the deflecting mode are unavoidable, its compact size and compatibility with the phase control system envisioned for CLIC [15] are promising. Where the high power waveguide forms part of an interferometer as is necessary for the accurate phasing of the CLIC crab cavities to $5 \mathrm{fs}$, then a single path to the input couplers reduces the error from unbalanced reflections as it could happen with dual feed. The monopole component of the deflecting mode can however reduce the accuracy of the phase control, requiring it to be eliminated or sufficiently reduced.

This paper discusses the excitation of multipole field components in the crab cavity by various couplers and possible solutions to minimize the monopole component. As an example of how these methods can be applied to real cavities, each solution will be compared with respect to overall performance for the first prototype of the CLIC crab cavity design.

\section{THE CLIC CRAB CAVITY}

In order to effectively demonstrate the methods used in this paper, the CLIC crab cavity is used as an illustration. The CST MICROWAVE STUDIO model of the example structure considered in this paper is shown in Fig. 1. The operating mode of $2 \pi / 3$ and frequency of $11.9942 \mathrm{GHz}$ are chosen identical as those of the main linac [16]. It is designed to be a 12 cell structure with racetrack cell shape for a frequency separation of $1 \mathrm{GHz}$ between the operating mode and the same order dipole mode (SOM). The SOM frequency of $13 \mathrm{GHz}$ gives less SOM dominated wakefield compared to a cylindrical cell shape [17]. An iris with diameter of $2 a=10 \mathrm{~mm}$ and thickness $2 \mathrm{~mm}$ is used giving a group velocity of $-2.92 \%$ and low short range wakefield. A key feature of the design which is not relevant to the present study is its insensitivity to dipole beamloading [16]. A peak power of $13.3 \mathrm{MW}$ is required to give a

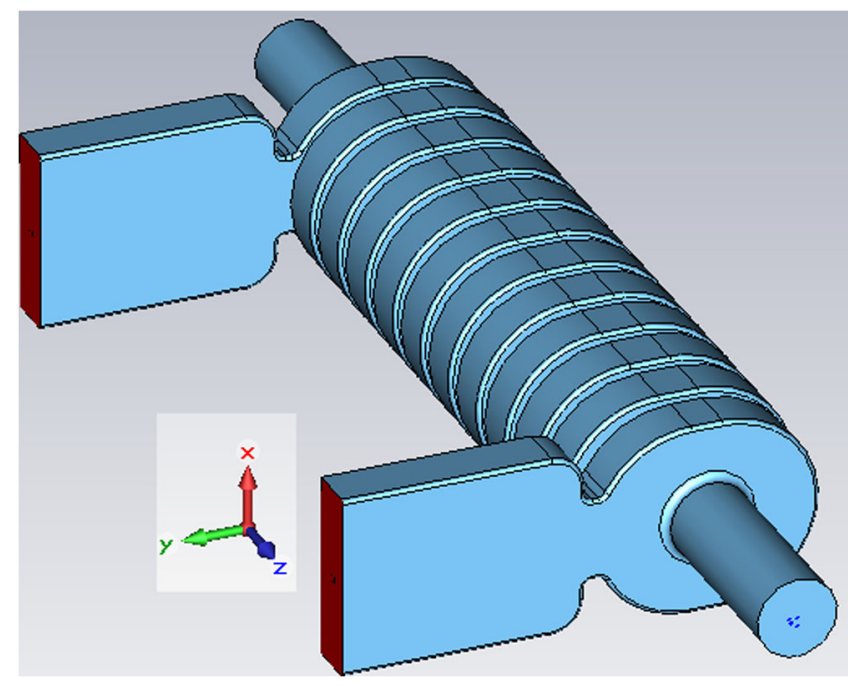

FIG. 1. MICROWAVE STUDIO model of the single-feed undamped crab cavity prototype for CLIC.

2.55 MV transverse kick as would be required to rotate the CLIC bunches at its top energy by $10 \mathrm{mrad}$ [6].

The coupler is matched to the cavity by adjusting the end cell radius and coupling slot dimensions. The phase advance and internal reflection calculated from the on-axis magnetic field by Kroll's method [18] are plotted in Fig. 2 and show an excellent match. For all geometric modifications discussed in later sections the structure was similarly
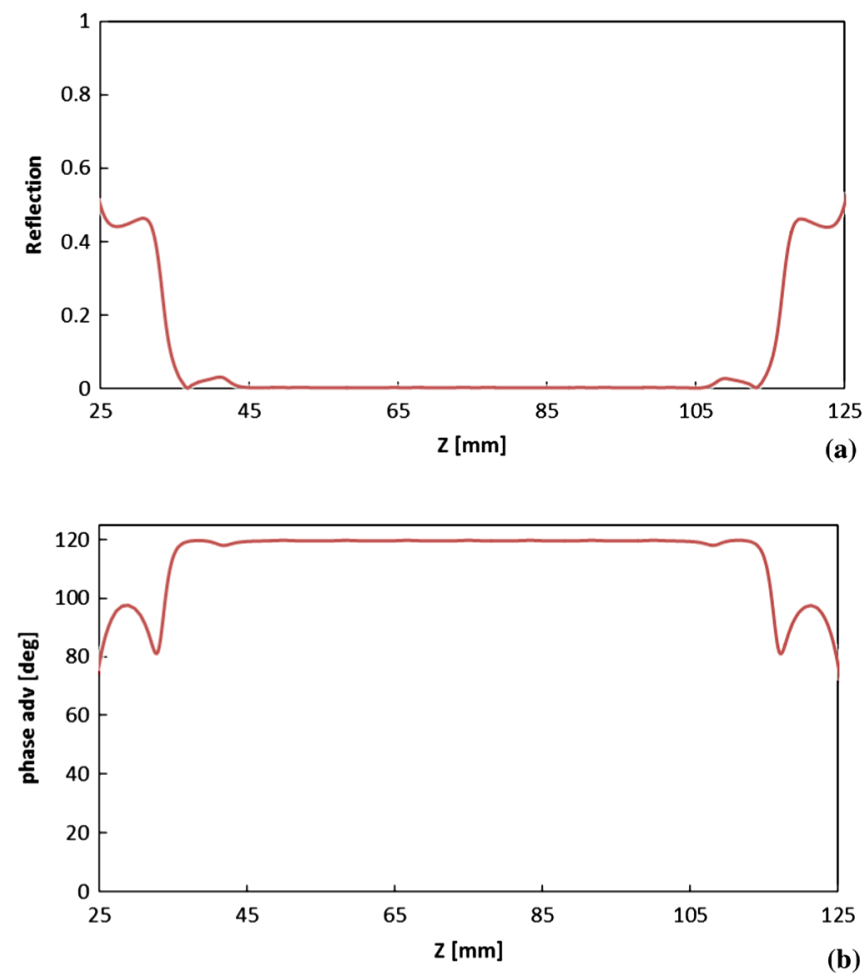

FIG. 2. Kroll's matching parameters calculated from the $B_{x}$ field for the crab cavity. (a) Internal reflection. (b) Phase advance per cell. 
well matched. Throughout the paper, unless otherwise stated in the figure captions, (i) all figures correspond to $11.9942 \mathrm{GHz}$ and a peak input power of $1 \mathrm{~W}$ at the input coupler port; (ii) all curves are drawn for the phase at which the beam is in quadrature to the deflecting voltage (the operating point for crab cavities); and (iii) the $X$ axis is vertical, the $Y$ axis is horizontal, and perpendicular to the beam direction $Z$. The beam is deflected horizontally.

In an accelerating cavity, the on-axis beam is accelerated longitudinally (in the $Z$ direction) by the longitudinal electric field $\left(E_{z}\right)$. For a deflecting cavity, the beam is kicked transversely by both the transverse electric field $\left(E_{y}\right)$ and transverse magnetic field $\left(B_{x}\right)$. The combined kick or transverse Lorentz force on a charge $q_{e}$, moving with a velocity $v_{z}$, can be expressed as

$$
F_{y}=q_{e}\left(E_{y}+v_{z} \times B_{x}\right)
$$

The in phase and quadrature forces (real and imaginary parts, respectively) due to the electric and magnetic fields that act on an electron traveling at the speed of light through the CLIC crab cavity are plotted in Fig. 3. For the iris diameter chosen, the force due to the magnetic field is about $50 \%$ higher than the force due to the electric field in the cell center. In the iris, the electric field kick is about twice the magnetic field kick. This is true only in the regular cells, as the kick is mainly magnetic in the end cells.
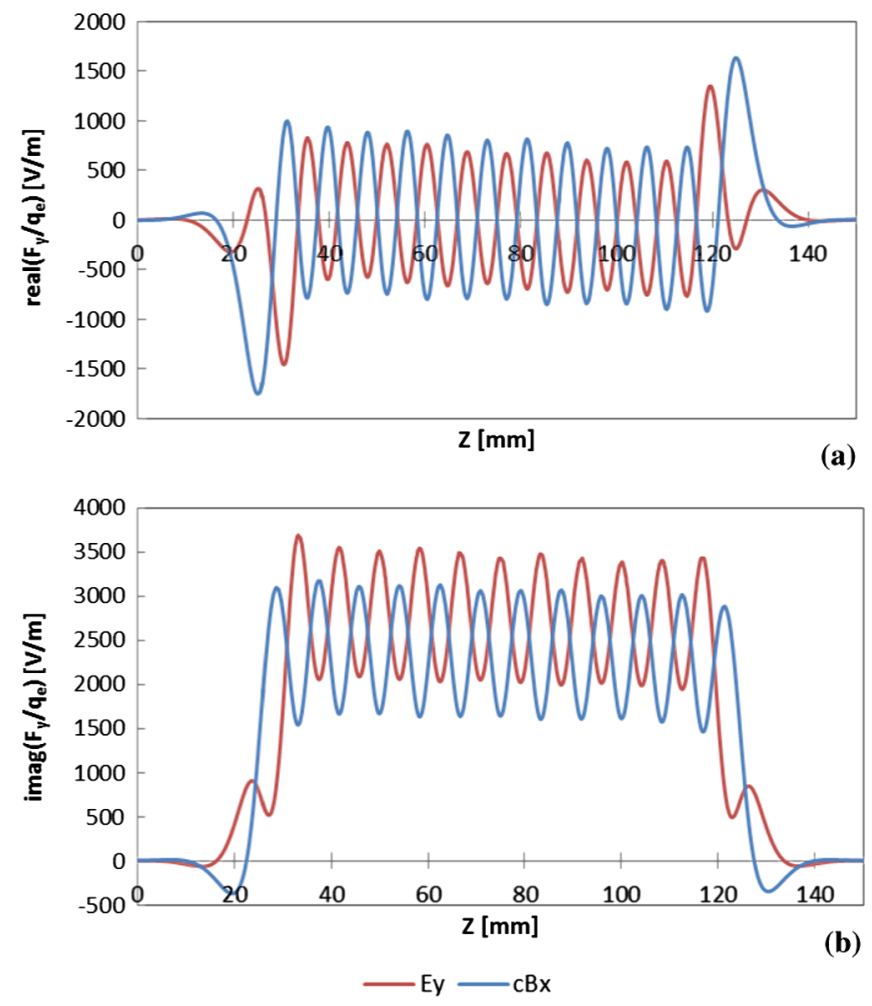

FIG. 3. Transverse electric and magnetic fields seen by the beam. (a) Real part. (b) Imaginary part.

\section{MULTIPOLE EXPANSION}

Geometric perturbations in an rf structure can introduce field asymmetries which can be expanded in terms of its multipole field components. The longitudinal electric field $E_{z}$ of the TM-like modes in a crab cavity can be expanded approximately in terms of radial and azimuthal variations as [19]

$$
E_{z}(r, \theta, z) \sim \sum_{m} E_{z m}(z) r^{m} \cos (m \theta) .
$$

As per Eq. (2), the lowest order $m=0$ pole component will accelerate or decelerate the beam.

Panofsky-Wenzel's theorem [20] relates the transverse kick to the longitudinal electric field as

$$
\begin{aligned}
V_{t} & =-i \frac{c}{\omega} \int_{0}^{L} \nabla_{\perp} E_{z}(r, \theta, z) d z \\
& =-i \frac{c}{\omega} \int_{0}^{L} \hat{r} \frac{\partial E_{z}(r, \theta, z)}{\partial r}+\hat{\theta} \frac{\partial E_{z}(r, \theta, z)}{r \partial \theta} d z \\
& =-i \frac{c}{\omega} m r^{m-1}\{\hat{r} \cos (m \theta)-\hat{\theta} \sin (m \theta)\} \int_{0}^{L} E_{z m}(z) d z .
\end{aligned}
$$

In accelerating cavities, among the multipoles, the dipole component is the most problematic while in deflecting cavities it is the monopole component. For the example crab cavity taken in this paper, the multipole components are calculated by using the fields along the longitudinal axis $(Z)$ at various azimuthal positions and at radial offset $r=0 \mathrm{~mm}$ for $m=0$ and at $r=1 \mathrm{~mm}$ for $m>0$ poles.

\section{INTERACTION BETWEEN THE MONOPOLE COMPONENT AND THE BEAM}

Beamloading in a general sense can be defined as the change in the electromagnetic fields of the operating mode due to the field induced or absorbed by the beam. In other words, it is a perturbation to the operating fields by the beam current. Assuming a bunch passing through the center of an ideal dipole mode, there is no beamloading. A bunch passing at a horizontal offset will result in dipole beamloading depending on the bunch parameters such as the charge, profile, and trajectory of the bunch and the rf parameters such as the frequency, amplitude, and phase of the operating mode. The coupler induced monopole component, which was previously shown to be a component of the total cavity field, thus becomes a component of the total beamloading. Here we consider effects only due to coupler asymmetries on the deflecting mode which we validate separately from longitudinal wakefields. For on-axis bunches, the beamloading from the monopole component of the operating mode can cause an energy change to the bunch. In the $\mathrm{TeV}$ machines like the CLIC, the resulting energy change for $1.5 \mathrm{TeV}$ beam is $6.16 \times 10^{-6} \%$, which is negligible compared to the tolerance of $0.1 \%$ [17], but in applications like photon sources or beam diagnostics the above energy spread can be significant. 


\section{COUPLER TYPES}

\section{A. Single-feed coupler}

In single-feed structures, there is a single waveguide or coaxial line coupled to the structure. An azimuthal asymmetry of the end cell or coupler cell is introduced by the single-feed coupler hence perturbs the cavity field. This perturbation can be represented by a multipole expansion allowing the required dipole field to be separated from terms with different azimuthal field variation. In addition to the dipole $(m=1)$ component, the single-feed coupler also introduces predominantly monopole $(m=0)$ and quadrupole $(m=2)$ components. As an example, the CLIC crab cavity has been simulated in CST MICROWAVE STUDIO and the multipole field components are extracted. For this structure, the multipole components of $E_{z}$, along the longitudinal axis are shown in Fig. 4, for a peak deflection voltage of $V_{\text {crab }}=$ $1 \mathrm{~V}$, up to $m=3$. The monopole component is located mainly in the end cells, while the dipole component is in all cells and is slightly higher in the regular cells. The fluctuations in the quadrupole and sextupole field strength are due to numerical noise of the simulation software. The location of the monopole field component in the end cells gives a clue for controlling the monopole field component without much effect on the dipole field. Integration of the above fields gives the complex $m$-pole voltage for $V_{\text {crab }}=1 \mathrm{~V}$ as follows: monopole, $-2.45 \times 10^{-2}-3.95 \times$ $10^{-6} \mathrm{iV}$; dipole, $251.38-1.22 \times 10^{-5} \mathrm{iV} / \mathrm{m}$; quadrupole,
$245.29-53.54 \mathrm{i} \mathrm{V} / \mathrm{m}^{2}$; and sextupole, $-6.89 \times 10^{4}-7.82 \times$ $10^{3} \mathrm{iV} / \mathrm{m}^{3}$. Note that the voltages are normalized with an offset of $r^{m}$, and are calculated at $r=1 \mathrm{~mm}$.

\section{B. Dual-feed coupler}

Using a dual-feed coupler retains the plane of transverse symmetry in both horizontal and vertical directions hence suppressing the monopole and quadrupole field components. The sextupole $(m=3)$ component is the lowest order of multipole field component present after the dipole component and is only present in the coupler cells [21]. A standard dual-feed coupler is shown in Fig. 5. As seen in the figure, the dual-feed structure is larger than the single feed. Note that this is a simplistic comparison because in practical short structures, flanges, bellows, wakefield dampers, and other associated hardware can dominate the size. For the CLIC crab cavity with dual feed, the multipole components extracted from CST MICROWAVE STUDIO for $V_{\text {crab }}=1 \mathrm{~V}$ are given as monopole: $1.32 \times 10^{-5}+3.02 \times$ $10^{-5} \mathrm{i} \mathrm{V}$, dipole: $251.38-3.58 \times 10^{-5} \mathrm{i} \mathrm{V} / \mathrm{m}$, quadrupole: $16.48+50.52 \mathrm{i} \mathrm{V} / \mathrm{m}^{2}$ and sextupole: $-1.02 \times$ $10^{5}+6.7 \times 10^{4} \mathrm{i} \mathrm{V} / \mathrm{m}^{3}$. The voltages are normalized with an offset of $r^{m}$, where $r=1 \mathrm{~mm}$. As the structure is symmetric, the magnitudes of monopole and quadrupole components should be zero, so monopole voltage below $3.5 \times 10^{-5} \mathrm{~V}$ and quadrupole voltage below $55 \mathrm{~V} / \mathrm{m}^{2}$ can be considered as contribution from numerical noise. The quadrupole component is $5.5 \times 10^{-5} \mathrm{~V}$ at $r=1 \mathrm{~mm}$
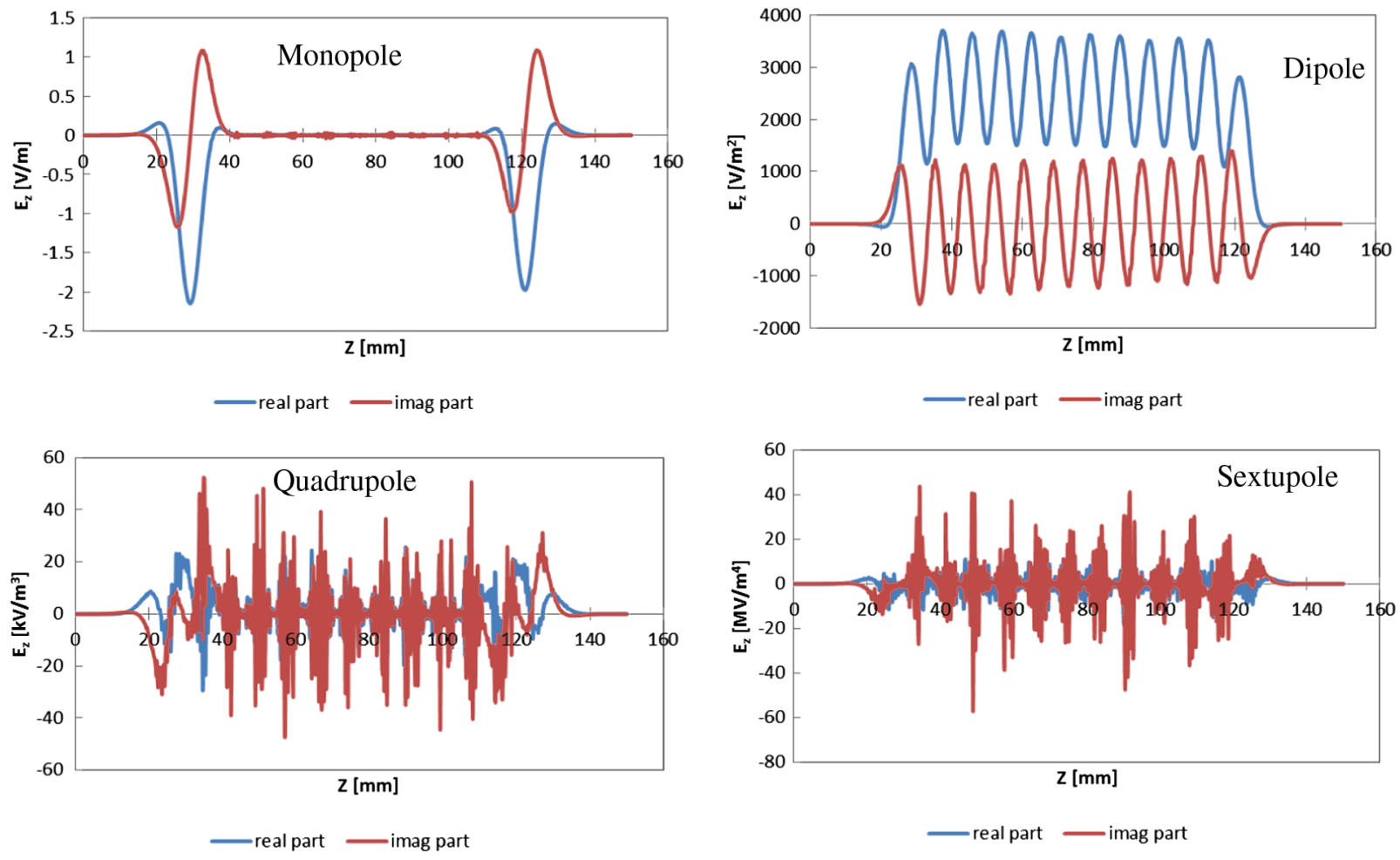

FIG. 4. Multipole components of the longitudinal electric field $E_{z}$ in the crab cavity for $V_{\text {crab }}=1 \mathrm{~V}$. 


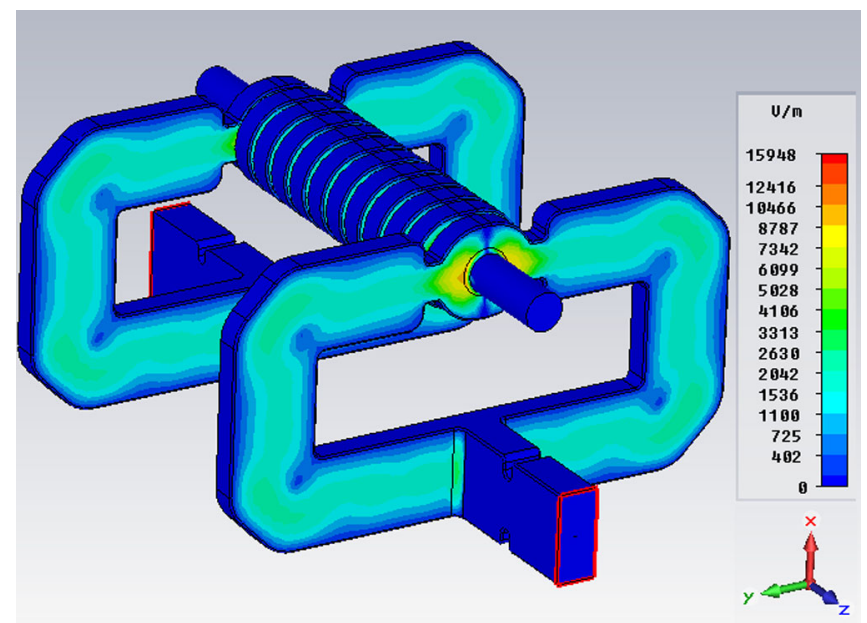

FIG. 5. Longitudinal electric field in the CLIC crab cavity with dual-feed coupler.

which is of the same order of magnitude as the monopole voltage. Hence, the noise levels in the dipole and sextupole components can also be approximated from a linear fit $\sim 4.5 \times 10^{-2} \mathrm{~V} / \mathrm{m}$ and $\sim 65 \times 10^{3} \mathrm{~V} / \mathrm{m}^{3}$, respectively.

\section{Single feed with dummy waveguide}

In standard single-feed structures, the $\mathrm{TE}_{10}$ mode of the rectangular waveguide couples to the $\mathrm{TM}_{110}$-like mode in the end cell of the structure in the horizontal plane. This geometrical asymmetry introduced by the coupler causes the field also to become asymmetric by shifting the electrical center of the dipole mode towards the coupler. This creates a real longitudinal electric field component on-axis which is in quadrature with the magnetic field. This field asymmetry can be completely corrected by using a dummy waveguide [22] thus eliminating the monopole component. There also exists a power flow asymmetry in the coupler cell as shown in Fig. 6. Most of the power flow comes in horizontally from the coupler and then bends round to the longitudinal direction down the structure; however, as the whole iris is fed from a single coupler there is a net power flow across the center of the cell in the horizontal direction. As the magnetic field of the deflecting mode is in the vertical direction, the above power flow requires a longitudinal electric field along the axis in phase with the magnetic field to provide a real horizontal Poynting vector. This asymmetry due to the power flow is more significant in traveling wave structures than in standing wave structures. The combination of the monopole components due to geometric asymmetry and the power asymmetry give a complex longitudinal electric field component. Using a dummy waveguide can eliminate the monopole component due to the mode offset and thus symmetrizes the field about the geometrical center. However, it does not symmetrize the power flow, hence, an imaginary longitudinal electric field component remains on axis. The dummy waveguide scheme is shown in Fig. 7 for the CLIC crab

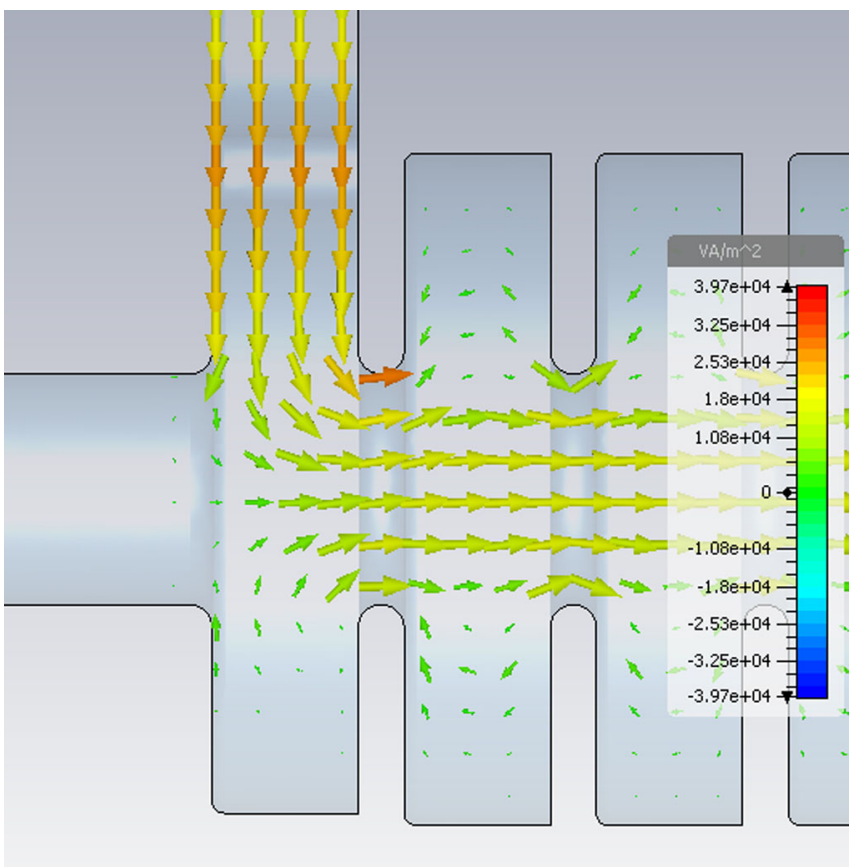

FIG. 6. Poynting vector in the $X Z$ plane for the CLIC crab cavity with single-feed coupler.

cavity. For a waveguide length higher than one wavelength, or $w g_{l}=67 \mathrm{~mm}$, the mode can be centered by adjusting the waveguide width $w g_{a}$. For $w g_{a}=10.375 \mathrm{~mm}$, the field is symmetric and the minimum occurs at the center of the cavity. However, due to the power flow asymmetry the monopole component is not zero, it is just out of phase with the dipole component. The multipole components for this structure are given as monopole: $3.45 \times 10^{-6}-2.32 \times$ $10^{-4} \mathrm{iV}$, dipole: $251.38-1.85 \times 10^{-4} \mathrm{iV} / \mathrm{m}$, quadrupole: $13.33+45.48 \mathrm{iV} / \mathrm{m}^{2}$ and sextupole: $-6.84 \times 10^{4}+9.28 \times$ $10^{3} \mathrm{iV} / \mathrm{m}^{3}$, all for a $V_{\text {crab }}=1 \mathrm{~V}$. Here the monopole component is reduced by 2 orders of magnitude however the phase between the monopole and dipole components seems to shift.

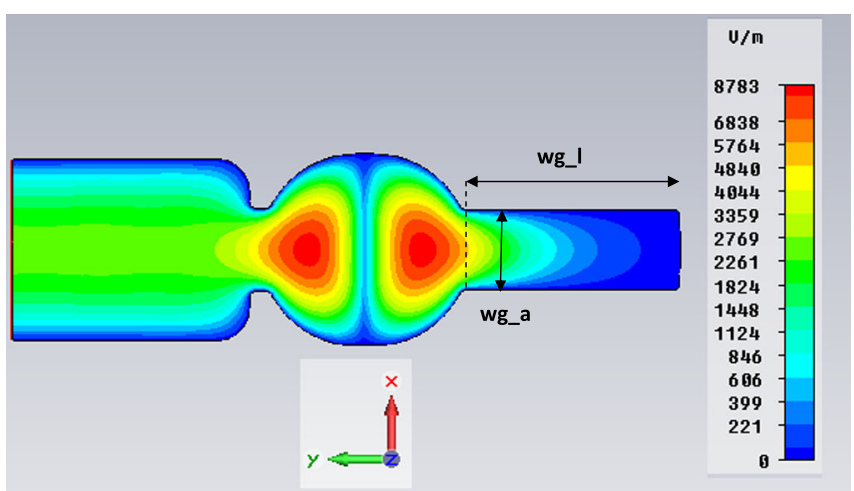

FIG. 7. Absolute value of longitudinal electric field in the end cell with dummy waveguide. 

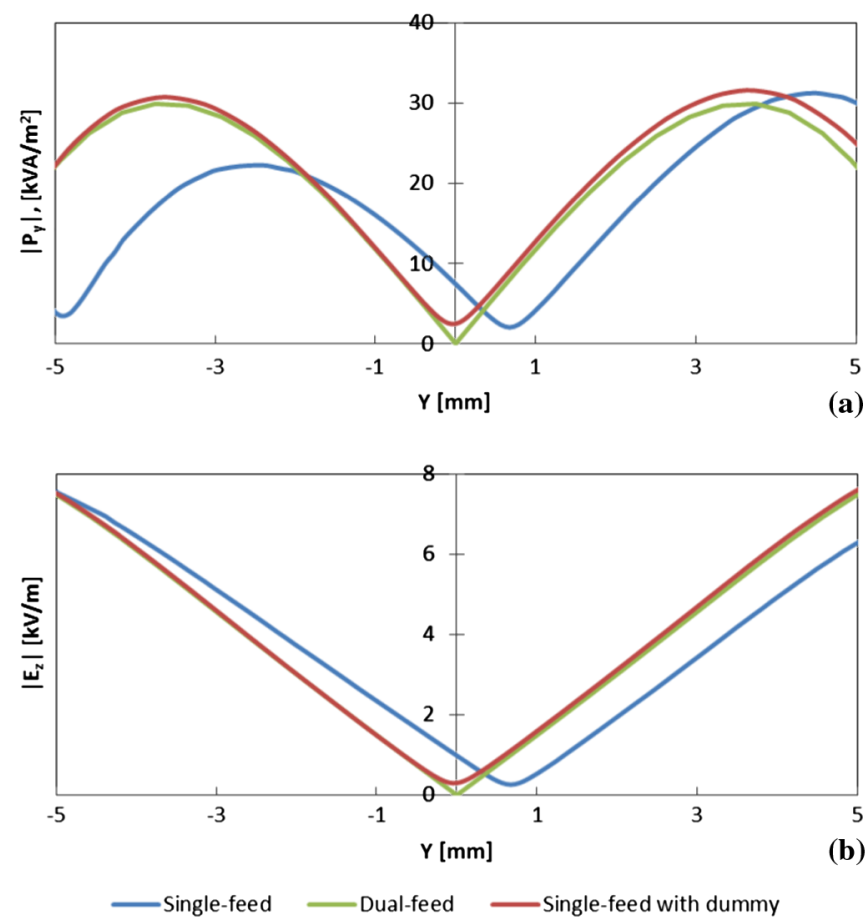

FIG. 8. Magnitude of (a) Poynting vector $P_{y}$ and (b) electric field $E_{z}$ on the $Y Z$ plane at $X=0$.

Magnitudes of the Poynting vector $\left(P_{y}\right)$ and longitudinal electric field $\left(E_{z}\right)$ along the horizontal direction $Y$ are plotted in Fig. 8 for the CLIC crab cavity with three different coupler types discussed above. The figure clearly shows that even with the dummy waveguide, the achievable monopole reduction is limited by the nonzero power flow across the axis.

\section{ROTATION OF INPUT AND OUTPUT COUPLERS}

If we alternate the direction of the input and output couplers such that one is on the left and the other on the right of the cavity, the phase difference of the monopole component flips by $180^{\circ}$ between the first and last cells. Assuming the walls are perfect electric conductors (PEC), mutual end cell rotation will compensate the acceleration at one end by the deceleration at the other end, thus canceling the real part of the monopole voltage. As explained in the previous section, there is an additional phase shift $\phi_{m d}$ between the dipole and monopole components in the coupler cells. If the monopole component is in phase with the dipole then the imaginary part $\sim \sin \left(\phi_{m d}\right)$ will be zero and hence the entire monopole component will be canceled.

Figure 9 shows the phase of the monopole and dipole components with respect to a relativistic beam in the CLIC crab cavity with PEC walls. The zero crossings of the phase correspond to the center position of each cell. It is clear that the asymmetric coupler has shifted the monopole phase from the dipole phase in the end cells showing the

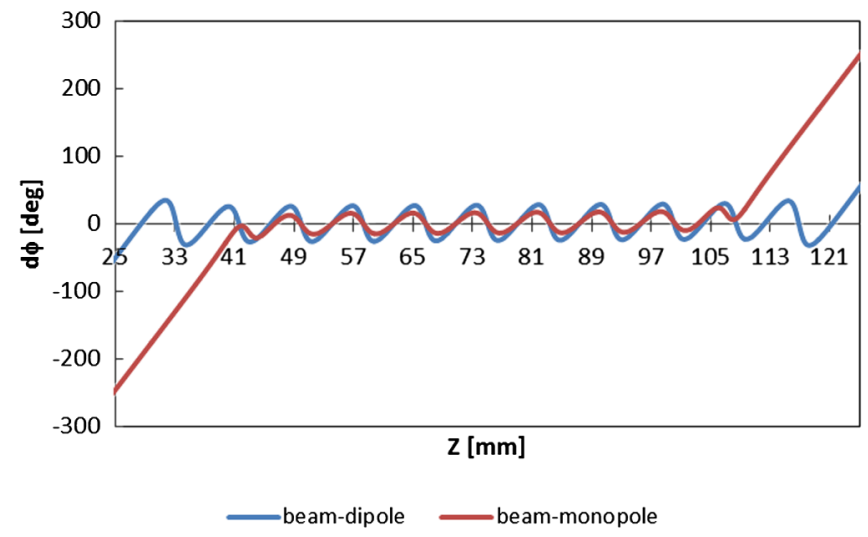

FIG. 9. Phase difference between beam and (a) dipole and (b) monopole components of $E_{z}$.

existence of a traveling wave perpendicular to the beam axis. This phase shift changes sign between the end cells due to the change in the direction of the power flow. Because of this $\phi_{m d}$, on-axis beamloading is complex in nature rather than purely real or imaginary.

The on-axis voltage due to the monopole field component can be expressed as

$$
V_{m}=\int_{0}^{L \text { cav }}\left|E_{z m}\right| \exp \left(\mathrm{i}\left\{\phi_{\text {beam }}-\phi_{m}\right\}\right) d z
$$

where $\left|E_{z m}\right|$ is the magnitude and $\phi_{m}$ is the phase of the monopole component as a function of $Z$. From Fig. 9, the monopole component is shifted with respect to dipole by an angle $\phi_{m d}=\phi_{d}-\phi_{m}$. Assuming the monopole field is zero everywhere except the end cells, Eq. (4) can be reduced to only consider the end cells of length $L_{\text {cell }}$ each. Sign + or - applies whether the couplers are feeding on the same side or opposite sides, respectively:

$$
\begin{aligned}
V_{m}= & \int_{0}^{L \text { cell }}\left(\left|E_{z m 1}\right| \exp \left(\mathrm{i}\left\{\phi_{\text {beam }}-\phi_{d}+\phi_{m d}\right\}\right) d z\right. \\
& \pm \int_{0}^{L \text { cell }}\left(\left|E_{z m 2}\right| \exp \left(\mathrm{i}\left\{\phi_{\text {beam }}-\phi_{d}-\phi_{m d}\right\}\right) d z\right.
\end{aligned}
$$

If the beam is phased with the dipole component, $\phi_{\text {beam }}=\phi_{d}$,

$$
\begin{aligned}
V_{m}= & \int_{0}^{L \text { cell }}\left(\left|E_{z m 1}\right| \exp \left(+\mathrm{i} \phi_{m d}\right) d z\right. \\
& \pm \int_{0}^{L \text { cell }}\left(\left|E_{z m 2}\right| \exp \left(-\mathrm{i} \phi_{m d}\right) d z\right.
\end{aligned}
$$

Assuming (1) both end cells have identical fields, $E_{z m 1}=$ $E_{z m 2}=E_{z m}$ and (2) both $E_{z m}$ and $\phi_{m d}$ are independent of $Z$, and (3) defining the voltage $V_{z m}=\left|E_{z m}\right| L_{\text {cell }}$, we obtain

$$
V_{m}=V_{z m} \exp \left(+\mathrm{i} \phi_{m d}\right) \pm V_{z m} \exp \left(-\mathrm{i} \phi_{m d}\right)
$$


Case 1.-When the couplers feed the same side, the terms in Eq. (7) are additive or

$$
V_{m}=\cos (\phi) \int_{0}^{L \text { cell }}\left|E_{z m}\right| d z=2 V_{z m} \cos \left(\phi_{m d}\right) .
$$

The imaginary voltage cancels between the cells and the real voltage doubles. For $\phi_{m d}=90^{\circ}$, the real part become zero within each end cell.

Case 2.-When the couplers feed opposite sides, the terms are subtractive or

$$
V_{m}=2 \mathrm{i} V_{z m} \sin \left(\phi_{m d}\right)
$$

The voltage is purely imaginary and $\phi_{m d}$ should be either 0 or $180^{\circ}$ for a complete cancellation.

For the CLIC crab cavity with PEC walls, the real and imaginary components of the on-axis electric field $\left(E_{z m}\right)$ seen by a relativistic beam are shown in Fig. 10 for the above two coupler alignment cases. For couplers on the same side, integration of the monopole field in Fig. 10(a) gives $V_{m}=$ $-2.37 \times 10^{-2}+8.54 \times 10^{-6} \mathrm{i} \mathrm{V}$ which is mainly real. When the couplers are alternated, the monopole component is $V_{m}=-7.07 \times 10^{-5}-3.42 \times 10^{-3} \mathrm{i} \mathrm{V}$, mainly imaginary and slightly smaller. Both voltages are given for a crabbing voltage of $V_{\text {crab }}=1 \mathrm{~V}$. In the former case, real parts add up and imaginary parts cancel between the cells, and vice versa in the latter.

However, if we consider a constant impedance structure with walls of finite conductivity, the field in the power flow
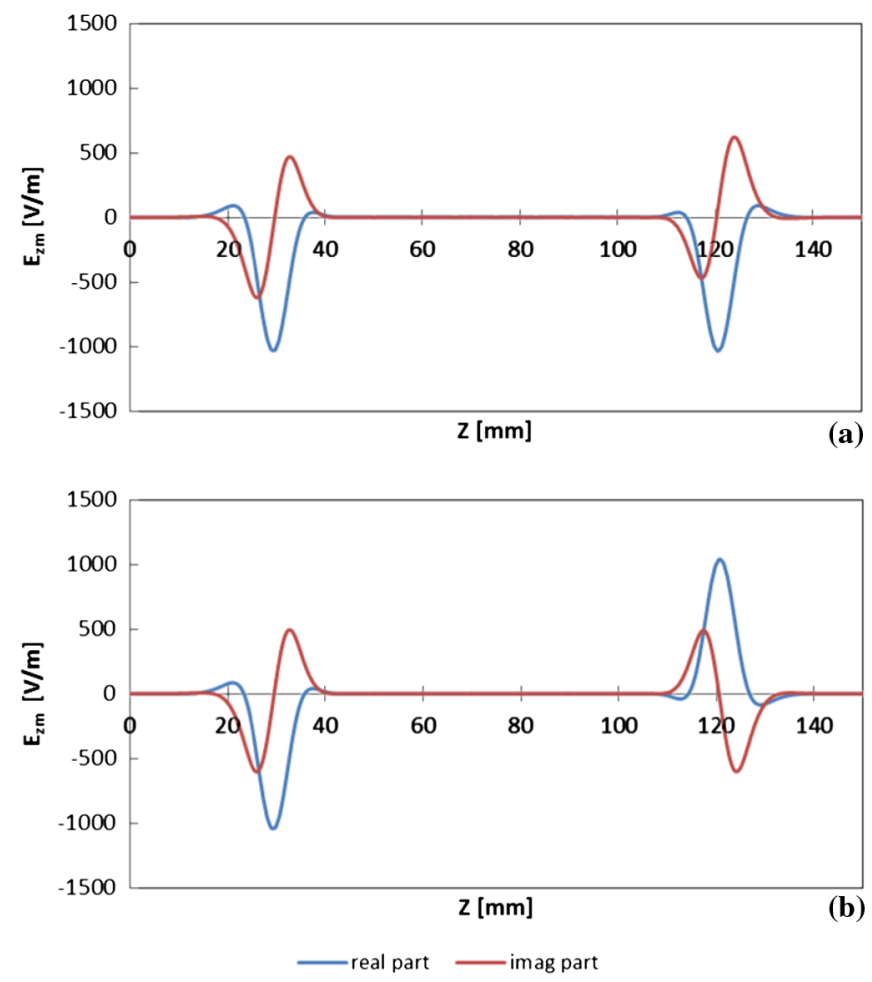

FIG. 10. On-axis electric field for the original structure when couplers feed (a) the same side and (b) opposite sides. downstream (output coupler) is always smaller than that in the upstream due to the attenuation of the fields caused by Ohmic losses. This introduces an amplitude imbalance in Eq. (7) as

$$
\begin{aligned}
V_{m} & =V_{z m} \exp \left(+\mathrm{i} \phi_{m d}\right) \pm n V_{z m} \exp \left(-\mathrm{i} \phi_{m d}\right) \\
& =V_{z m}\left\{(1+n) \cos \left(\phi_{m d}\right) \pm i(1-n) \sin \left(\phi_{m d}\right)\right\} .
\end{aligned}
$$

Cancellation requires the individual terms of Eq. (11) to be zero which is impossible when $n \neq 1$ although either the real or imaginary term can still be canceled by setting $\phi_{m d}=0^{\circ}$ or $90^{\circ}$. For the 12 cell CLIC structure, $n=$ 0.93. Now for case 1 and case 2 of the CLIC structure simulated with copper walls, the respective monopole voltages are given by $V_{m}=-2.45 \times 10^{-2}-3.95 \times$ $10^{-6} \mathrm{i} \mathrm{V}$ and $V_{m}=-1.05 \times 10^{-3}+1.9 \times 10^{-3} \mathrm{i} \mathrm{V}$. To compare the lossless and lossy cases, it is worth noting in Fig. 10 that in the end cell, the real part of the electric field is mainly one sided while the imaginary part is oscillatory. Hence, the integrated imaginary voltage is much smaller than the real voltage per cell. Because of this, there is no big difference between the lossless and lossy structures, when the couplers feed the same side but a significant difference in the real part when couplers feed opposite sides.

\section{MINIMIZATION OF THE MONOPOLE VOLTAGE}

The energy change to a single bunch due to the monopole component of the deflecting mode can be reduced as explained in Sec. VI by using global compensation where the total energy change of the bunch is reduced to zero when the bunch transits through the structure. However, perfect cancellation is impossible as the fields in the end cells are different due to losses, deviation of rf field in the design, and longitudinal asymmetries within the cell. Two methods to correct the longitudinal asymmetries within the end cell are discussed below.

\section{A. End cell length adjustment}

In structures with beampipe, there exists a longitudinal asymmetry in the end cell about its center. This causes the cell to have different coupling capacitance on either side resulting in an asymmetry in the field. This differential capacitive loading effect is much less in small beampipe structures $(2 a \ll \lambda)$ than for the CLIC crab cavity, $2 a=$ $0.4 \lambda$. From Fig. 10(b), when the couplers are alternated, both the real and imaginary parts of the monopole voltage are supposed to flip by $180^{\circ}$. But this only happens perfectly with the real parts of the field which then cancel each other on integration. The imaginary part does not undergo the flip because of the in-cell asymmetry of the field. Minimization of the imaginary part requires longitudinal 


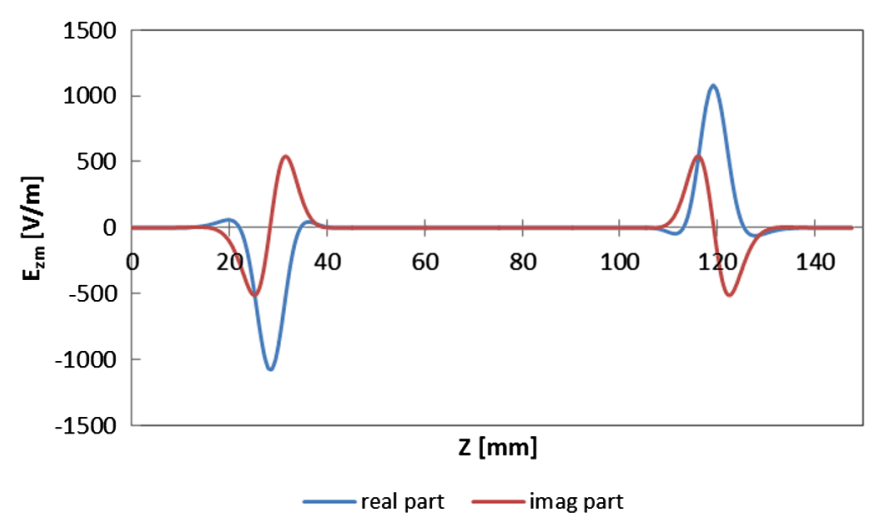

FIG. 11. On-axis electric field for reduced end cell length $L_{\text {end }}=7.125 \mathrm{~mm}$.

in-cell symmetrization by adjustment of the beam phase in the end cells. This can be done by changing the end cell length which will add an extra phase $\Delta \phi$ in Eq. (7) to the exponent to make it $\phi_{m d}+\Delta \phi$. For CLIC, the extra phase shift required to make the sine function in Eq. (9) zero is estimated as $\sim 16^{\circ}$. Corresponding end cell length is $L_{\mathrm{end}}=$ $7.125 \mathrm{~mm}$ (nominally $8.332 \mathrm{~mm}$ ). The resulting monopole field is shown in Fig. 11 and the corresponding voltage is $V_{m}=1.59 \times 10^{-5}+8.94 \times 10^{-6} \mathrm{iV}$, for $V_{\text {crab }}=1 \mathrm{~V}$ with PEC. Table I compares the monopole and quadrupole components with and without the end cell length change for the above-mentioned CLIC structure with PEC walls. With losses added, the required phase shift is estimated as $\Delta \phi \sim 9^{\circ}$ which is achieved by an end cell length of $L_{\text {end }}=7.825 \mathrm{~mm}$. The resulting monopole voltage is $1.02 \times 10^{-3}-1.18 \times 10^{-4}$ i. Here, the longitudinal symmetrization has not worked properly because of the field asymmetry between the end cells due to losses.

\section{B. Dummy waveguide}

It was discussed in Sec. V C that a dummy waveguide can be used to center the dipole mode, however it also causes a phase shift between monopole and dipole components as well. We can adjust the dimensions of the dummy waveguide in order to have the correct phase shift as well as symmetrizing the fields. In order to do this, the cutoff frequency of the dummy waveguide needs to be adjusted by changing its width $w g_{a}$. For CLIC crab cavity, the difference in phase between the monopole and the dipole

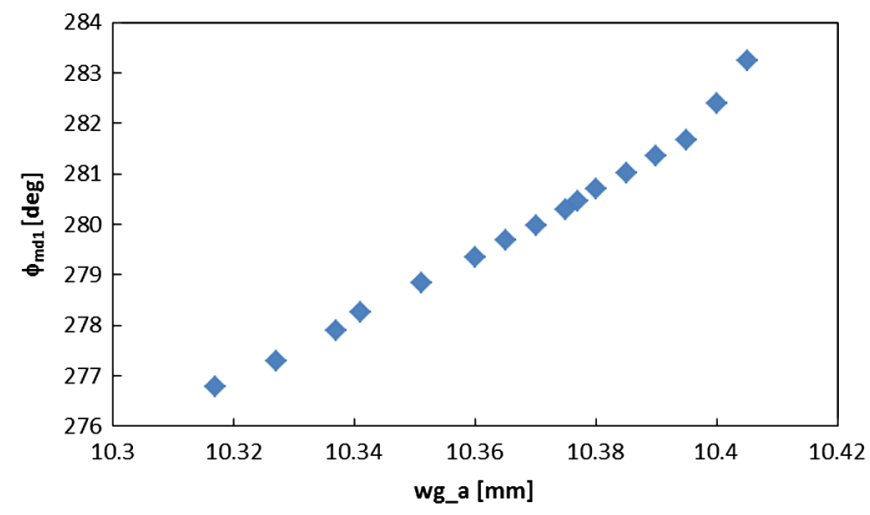

FIG. 12. Phase difference between the monopole and dipole components of the electric field in the middle of end cell $\left(\phi_{m d 1}\right)$ as a function of the dummy waveguide width $w g_{a}$.

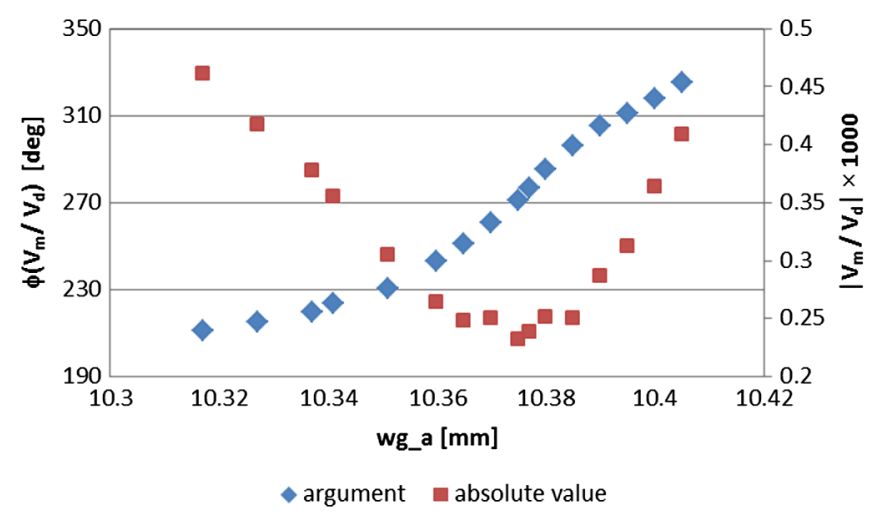

FIG. 13. Dependence of magnitude and phase of monopole voltage relative to dipole voltage on the dummy waveguide width $w g_{a}$, for $w g_{l}=67 \mathrm{~mm}, V_{\text {crab }}=1 \mathrm{~V}$.

components of $E_{z}$ in the middle of the end cell $\left(\phi_{m d 1}\right)$ varies with $w g_{a}$ as shown in Fig. 12. The couplers are at $0^{\circ}$ with respect to each other. Figure 13 shows the dependence of the amplitude and phase of the monopole voltage relative to the dipole, on $w g_{a}$. For $w g_{l}=67 \mathrm{~mm}$ and $w g_{a}=10.375 \mathrm{~mm}$, the amplitude minimum occurs at a phase of $\phi_{m d} \sim 270^{\circ}$ which is $3.45 \times 10^{-6}-2.32 \times$ $10^{-4} \mathrm{i}$ V. The amplitude cannot be adjusted to zero due to the required power flow across the cell. Table II compares the monopole and quadrupole components for

TABLE I. Monopole and quadrupole components for CLIC crab cavity structures discussed. All values correspond to PEC walls and $V_{\text {crab }}=1 \mathrm{~V}$.

\begin{tabular}{lccr}
\hline \hline Structure & Coupler alignment, deg & Monopole, $\left[\mathrm{V} / \mathrm{m}^{0}\right]$ & \multicolumn{1}{c}{ Quadrupole, $\left[\mathrm{V} / \mathrm{m}^{2}\right]$} \\
\hline Single-feed- & 0 & $-2.37 \times 10^{-2}+8.54 \times 10^{-6} \mathrm{i}$ & $2.35 \times 10^{2}-6.25 \times 10^{-1} \mathrm{i}$ \\
normal end cell & 180 & $-7.07 \times 10^{-5}-3.42 \times 10^{-3} \mathrm{i}$ & $7.06 \times 10^{-1}+2.51 \times 10^{1} \mathrm{i}$ \\
Single-feed- & 0 & $-2.50 \times 10^{-2}-7.10 \times 1 \mathrm{e}^{-6} \mathrm{i}$ & $2.33 \times 10^{2}-4.69 \times 10^{-1} \mathrm{i}$ \\
short end cell & 180 & $1.59 \times 10^{-5}+8.94 \times 10^{-6} \mathrm{i}$ & $-1.53 \times 10^{-1}-1.01 \times 10^{1} \mathrm{i}$ \\
\hline \hline
\end{tabular}


TABLE II. Effect of losses on the monopole and quadrupole components at $V_{\text {crab }}=1 \mathrm{~V}$ for the CLIC crab cavity with dummy waveguide.

\begin{tabular}{lccc}
\hline \hline Material & Coupler alignment, deg & Monopole $\left[\mathrm{V} / \mathrm{m}^{0}\right]$ & Quadrupole $\left[\mathrm{V} / \mathrm{m}^{2}\right]$ \\
\hline PEC & 0 & $5.83 \times 10^{-6}+2.14 \times 10^{-6} \mathrm{i}$ & $2.97-1.78 \times 10^{-2} \mathrm{i}$ \\
& 180 & $-2.26 \times 10^{-5}-7 \times 10^{-3} \mathrm{i}$ & $7.27 \times 10^{-2}+6.51 \times 10^{1} \mathrm{i}$ \\
Copper & 0 & $3.45 \times 10^{-6}-2.32 \times 10^{-4} \mathrm{i}$ & $1.33 \times 10^{1}+4.55 \times 10^{-1} \mathrm{i}$ \\
& 180 & $-3.98 \times 10^{-5}-6.99 \times 10^{-3} \mathrm{i}$ & $8.24 \times 10^{-1}+1.08 \times 10^{2} \mathrm{i}$ \\
\hline \hline
\end{tabular}

the structure with dummy waveguide with and without wall losses.

\section{BEAMLOADING MINIMIZATION}

In addition to experiencing an energy change, the bunch can also load the rf hence modify the amplitude and phase of the deflecting field seen by the succeeding bunches which can be a more serious issue. A change in amplitude will affect the rotation or kick given to the bunch leading to a small crossing angle or being deflected at the wrong angle. A phase change will result in an additional center of mass kick to the bunch. For CLIC, the amplitude tolerances on the crab cavity are quite loose $(2 \%)$ as a small change in crossing angle has little effect on the luminosity. The phase tolerance is much tighter as a small transverse offset at the IP could significantly reduce the luminosity due to the very small bunch sizes compared to the deflection of the particles in the head and tail of the bunch, hence the phase tolerance is only 19 mdeg. However, as many other effects will contribute to the amplitude and phase variations, the contribution from the monopole beamloading should be much smaller than the above numbers. Another typical crab cavity specification is given for the Argonne APS deflecting cavities, the required amplitude stability between the two cavities is $1 \%$ for $10 \%$ emittance variation and the phase stability is $80 \mathrm{mdeg}$ for $10 \%$ orbit distortion [23].

For cancellation of the kick between the first and last cells, the beam will locally add power to one cell and remove it from the other, hence the total energy balance will remain constant. However, for a backward traveling wave structure like the CLIC crab cavity, as time advances the downstream cell will have its power removed by the coupler, while the upstream end will have its power travel downstream. For the CLIC, the bunch spacing $(\sim 0.5 \mathrm{~ns})$ being a small fraction of the cavity fill time $(\sim 11.5 \mathrm{~ns})$, the resulting local perturbation in the deflecting field travels down the cavity at the group velocity of the structure. This will cause a variation in the deflecting voltage for a time equal to the fill time of the structure causing kick errors for the following bunches. While the beam would not actually provide any power to the structure, it would redistribute the fields such that the ratio of input power to output power would be altered, thereby altering the energy in the structure. For the CLIC crab cavity, about 23 bunches will see a varying deflecting amplitude and phase along the structure. Thus, for the single-feed crab cavity, in addition to reducing the monopole component over the full structure globally, it is also important to reduce the beamloading effect.

The steady state variation in deflecting voltage phase and amplitude can be approximated by calculating the beam induced power in the upstream end cell and adding it as a perturbation to the input power. This neglects the effect of the beam in the downstream cell, but since this power does not propagate down the structure its effect is small. The total loss for a traveling wave structure can be expressed as the sum of the power flowing through the structure, the power dissipated on the walls and the monopole beamloading as in Eq. (12). The unloaded input power $P_{\text {in }}$ is equal to the sum of the first two terms as

$\hat{P_{\text {loss }}}=P_{\text {flow }}+P_{\text {wall }}+P_{\text {beam }}=P_{\text {in }}+I_{\text {beam }} \times \hat{V_{\text {beam }}}$.

Depending on the phase shift of the monopole component with respect to the dipole component, the total loss can be either real or complex. A real loss will give rise to only amplitude variation and a complex loss to both amplitude and phase variation of the deflecting field as per Eq. (5):

$$
V_{\mathrm{def}}=\sqrt{\hat{P_{\text {loss }}} \times(R / Q) \times Q_{L}}
$$

where $R / Q$ is the transverse shunt impedance and $Q_{L}$ is the loaded $Q$ ignoring the beamloading. For the CLIC crab cavity, the unloaded input power is $13.3 \mathrm{MW}, R / Q$ is $6.06 \mathrm{k} \Omega / \mathrm{m}$, and $Q_{L}$ is 805 . For the single-feed structure, the monopole and dipole components are off by about $0^{\circ}$ or $180^{\circ}$ depending on the coupler orientation. For this example we choose the $180^{\circ}$ case. The beam current being $1.19 \mathrm{~A}$, then this monopole field component of the deflecting mode in the upstream end cell will give rise to a deflecting voltage with an amplitude change of $0.12 \%$ and a phase change of $7.2 \mathrm{mdeg}$, from the unloaded voltage of $2.55 \mathrm{MV}$. In the structure with dummy waveguide, the phase shift between the field components is about $90^{\circ}$. In this case the amplitude change is $0.008 \%$ and a phase change is $23.7 \mathrm{mdeg}$. These phase errors are much higher than the tolerance for the CLIC crabbing operation. As many other factors will also contribute to the phase error such as the phase shifts in distribution system, it is 


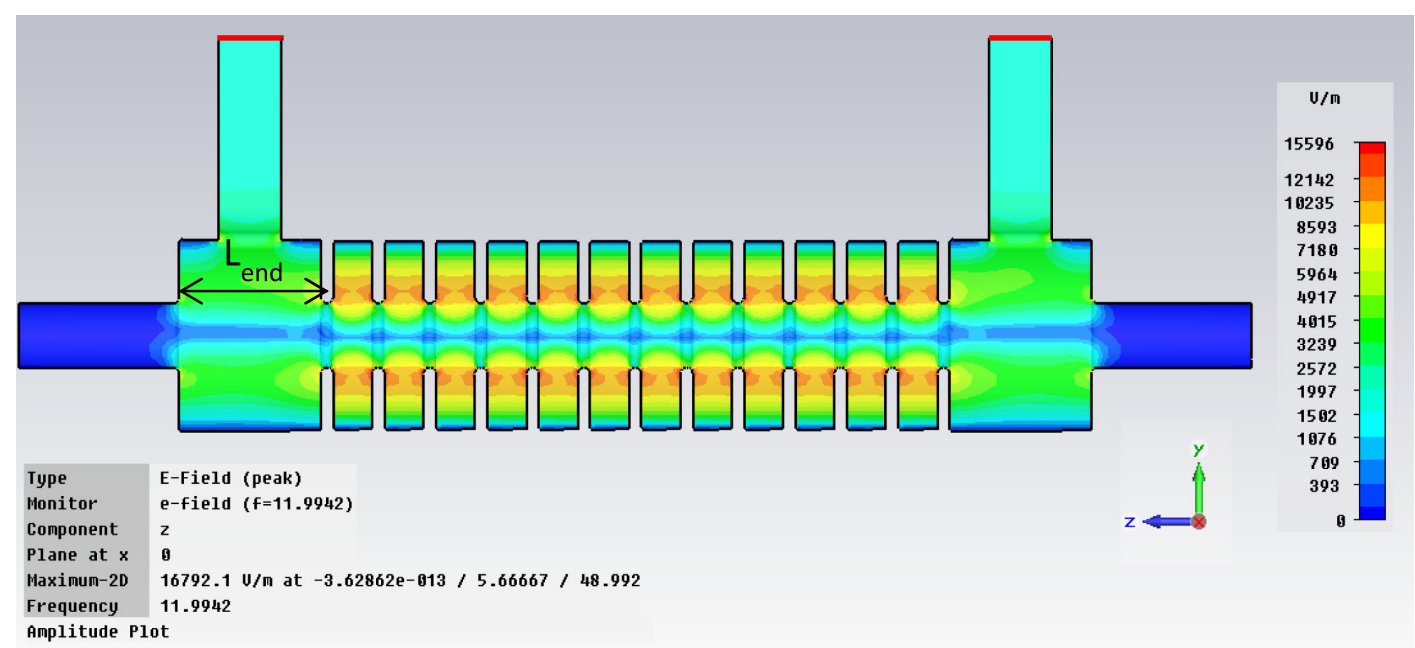

FIG. 14. Single-feed standard coupler with extra-long end cells.

preferred to have the field instability due to beamloading an order of magnitude lower than the above tolerances. If the amplitude of the monopole component is reduced by a factor 10 , then the amplitude and phase errors are also lowered by the same amount. It should be noted that the amplitude and phase of the monopole component of the deflecting mode depend only on the coupler cell geometry (which is fixed), the bunch charge and the bunch arrival time but not on the beam transverse position (which can jitter). Hence, its contribution to the beamloading can possibly be predicted and corrected by using a feed forward control. However, bunch to bunch charge and arrival time fluctuations will reduce the accuracy of the feed forward unless the monopole component is well minimized. An estimate of the resulting phase error would be $20 \mathrm{mdeg} /$ coulomb for the single-feed structure with dummy waveguide. However, this aspect needs thorough investigation which is not discussed here.

To avoid the issues associated with the monopole component altogether, dual-feed couplers have been considered as the baseline option. These however require a splitter which could complicate the CLIC phase control system

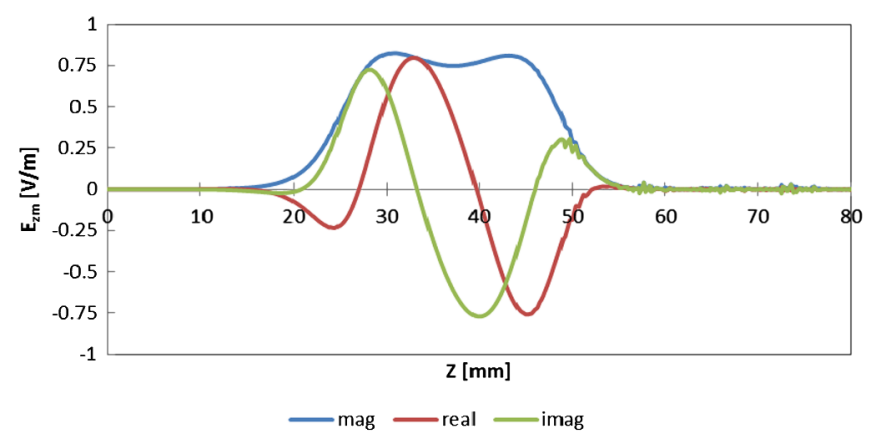

FIG. 15. On-axis electric field in the end cell showing cancellation within the cell for $V_{\text {crab }}=1 \mathrm{~V}$. and interferometer as mentioned in the end of Sec. I. Monopole-free single-feed couplers have been investigated as a potential simplification. This requires local monopole component cancellation in each of the end cells. As discussed previously, the power flow must also be symmetrized to avoid longitudinal on-axis electric fields. This means that either the longitudinal field must be canceled

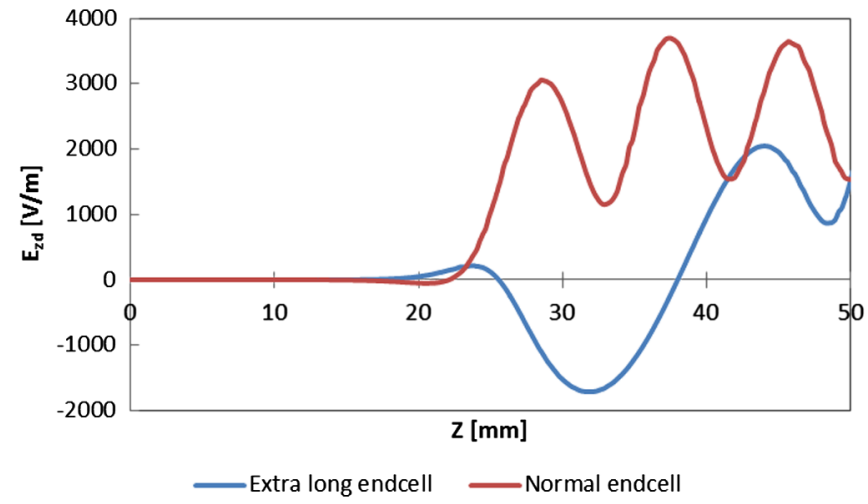

FIG. 16. Real part of the dipole field in the structure end section with and without extra-long cell for $V_{\text {crab }}=1 \mathrm{~V}$.
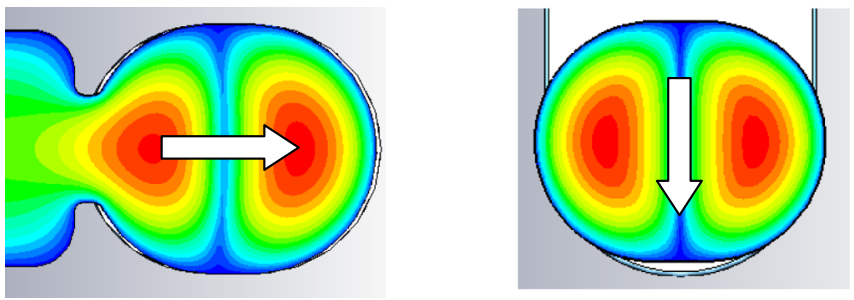

(a)

(b)

FIG. 17. Longitudinal electric field (a) $\mathrm{TE}_{10}$ coupled horizontally and (b) $\mathrm{TE}_{20}$ coupled vertically to the dipole mode. Arrow shows the direction of power flow. 


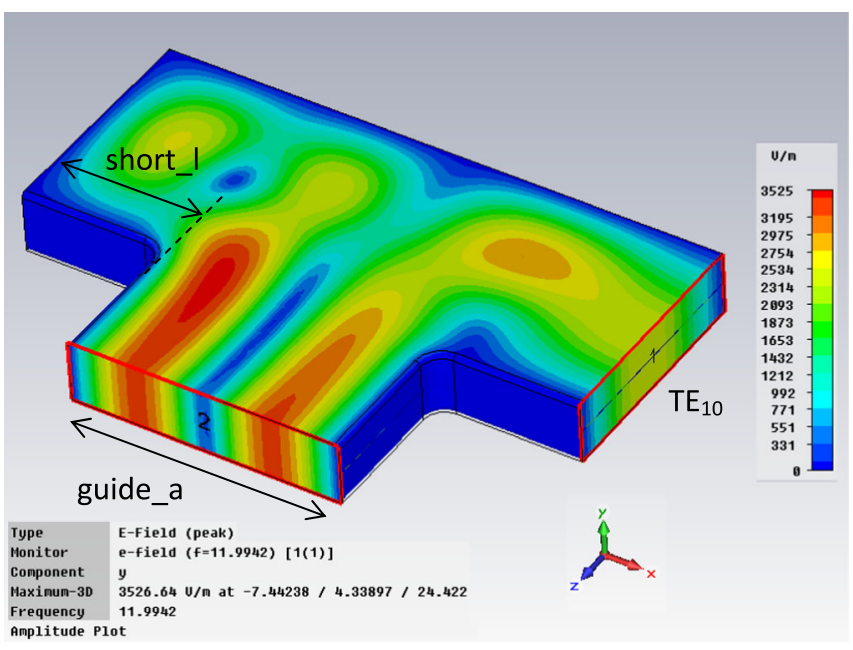

FIG. 18. Absolute $E_{y}$ field in a $\mathrm{TE}_{10}$ to $\mathrm{TE}_{20}$ mode converter at $11.9942 \mathrm{GHz}$ for matching parameter $L_{\mathrm{end}} / L_{\mathrm{reg}}=2.902$.

in a single cell or the power feed must be symmetric. Methods of achieving both are investigated.

\section{A. Using extra-long end cell}

By using end cells which are about 3 times longer than the regular cell $\left(L_{\text {reg }} \sim 2 \pi / 3\right)$, the beam will go through a full wave change of fields in the end cells. In that case, the real and imaginary parts of the monopole field will cancel within the cell itself. Because of the field asymmetry in the end cell due to the beampipe, the cell length has to be reduced from the nominal length of $L_{\text {end }} / L_{\text {reg }}=3$ to 2.902 for monopole cancellation. Such a design for the CLIC crab cavity is shown in Fig. 14 and the fields in one end cell in Fig. 15 for $V_{\text {crab }}=1 \mathrm{~V}$. For copper walls, the monopole voltage for $V_{\text {crab }}=1 \mathrm{~V}$ is $-1.48 \times 10^{-5}-$ $3.54 \times 10^{-5} \mathrm{i} \mathrm{V}$, which is around the required field reduction level. This can be further reduced by using a dummy waveguide as described in Fig. 7. In addition to the obvious disadvantage of the extra complexity added to the coupling cell, and possible trapped modes in the extended cell, the dipole kick in the end cells is also reduced by about $40 \%$ compared to the structure with nominal end cell length as shown in Fig. 16. Although this type of coupler solves the problem of monopole kick, practical use of it may depend on the specific application.

\section{B. $\mathrm{TE}_{20}$ coupler}

As previously mentioned, a traveling wave deflector with single-feed couplers suffers from both geometric and power flow asymmetries in the end cells. The geometric asymmetry can be negated with the use of a dummy waveguide, however power flow asymmetry cannot be canceled in this manner. As a way to overcome this fundamental limitation of traveling wave deflectors, a novel

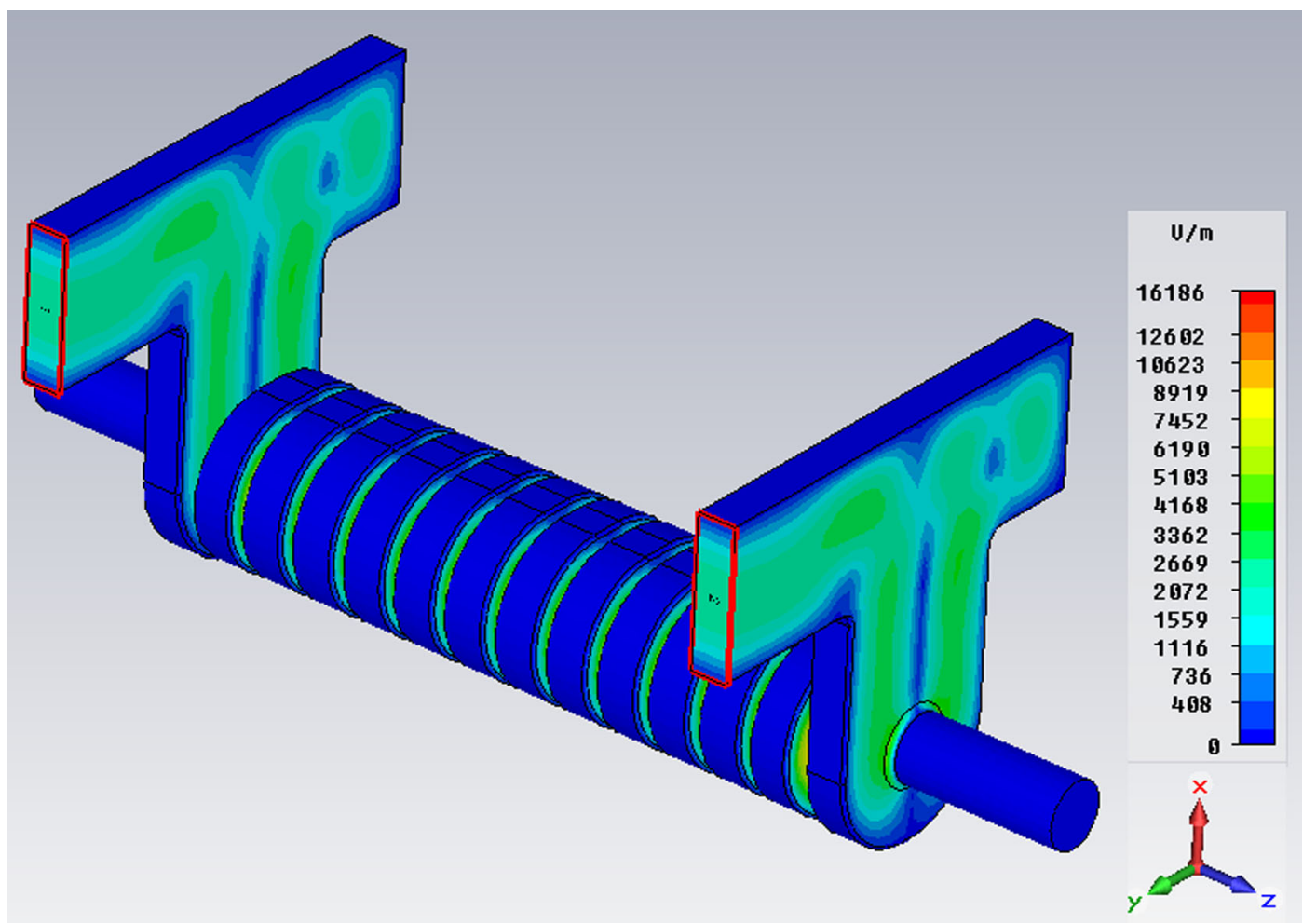

FIG. 19. Single-feed standard coupler with $\mathrm{TE}_{10}$ to $\mathrm{TE}_{20}$ adapter. 

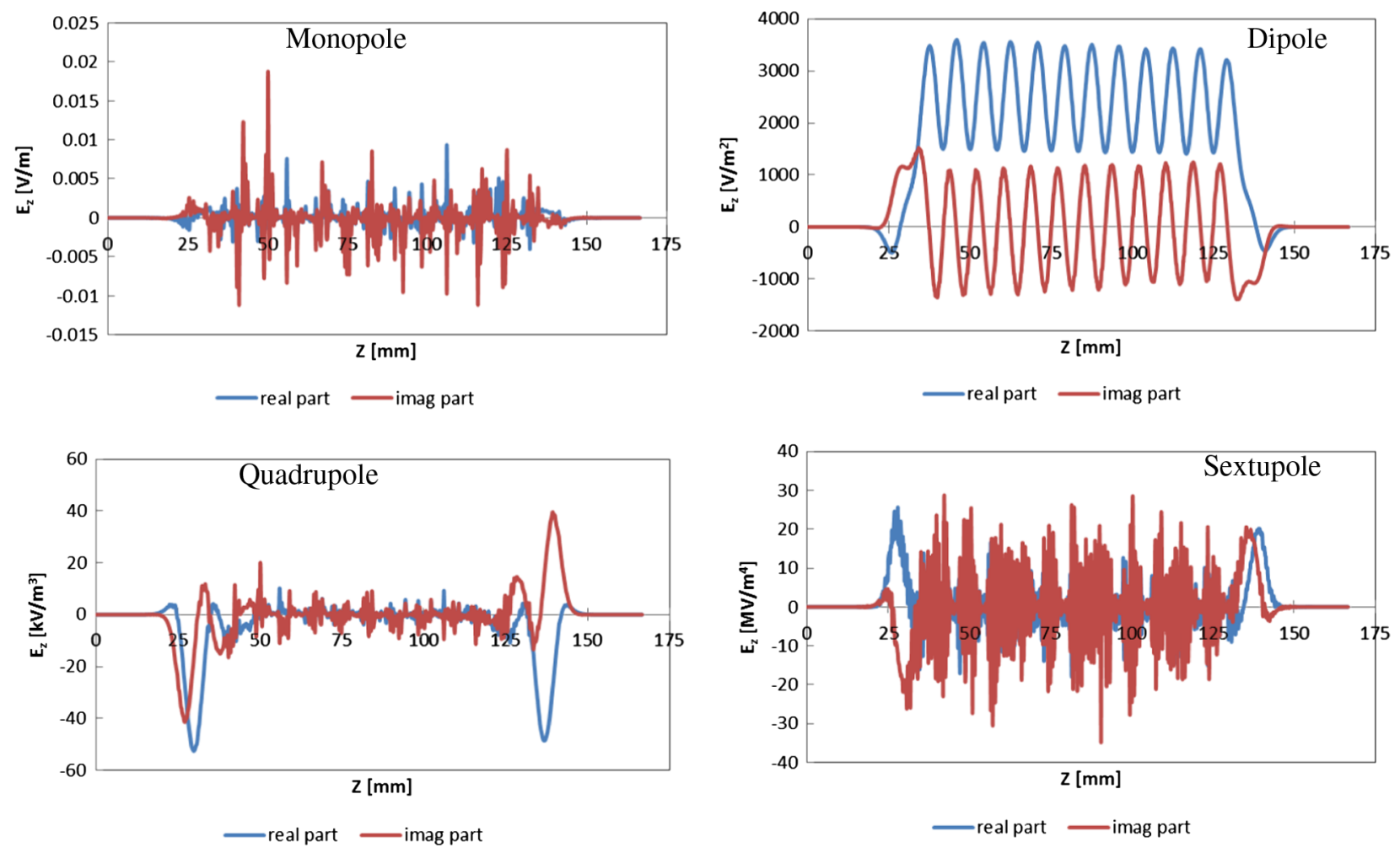

FIG. 20. Multipole components in $\mathrm{TE}_{20}$ coupled cavity for $V_{\text {crab }}=1 \mathrm{~V}$.
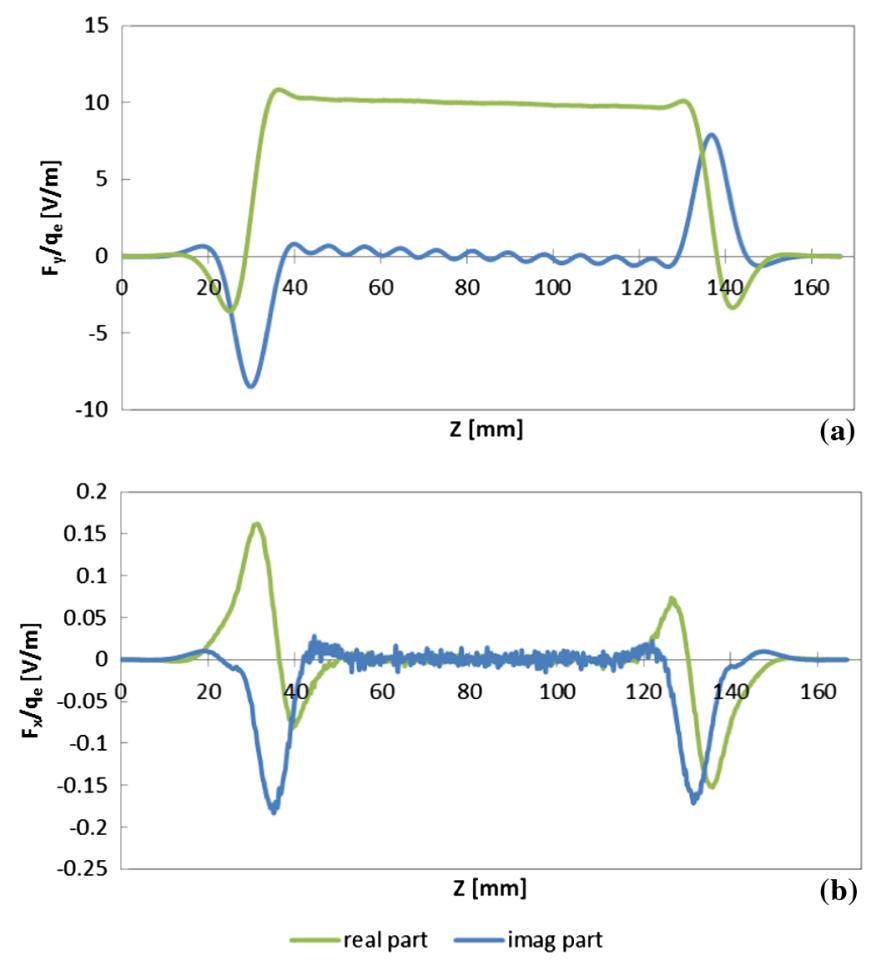

FIG. 21. (a) Horizontal and (b) vertical kicks for the $\mathrm{TE}_{20}$ coupled cavity for $V_{\text {crab }}=1 \mathrm{~V}$.

coupler is proposed based on the use of the $\mathrm{TE}_{20}$ mode of a rectangular waveguide to couple into the $\mathrm{TM}_{110}$-like mode of the cavity in the vertical plane. In this case the power flow will be in the vertical plane with a zero monopole component of the deflecting mode as shown in Fig. 17. If properly designed, this type of coupler can reduce the magnitude of monopole kick to zero. This will primarily need a $\mathrm{TE}_{10}$ to $\mathrm{TE}_{20}$ mode converter, a design for $11.9942 \mathrm{GHz}$ is shown in Fig. 18. In the design, length of the short plane, short $l$ and width of the $\mathrm{TE}_{20}$ waveguide, guide $a$ are the matching parameters. The mode converter should be designed to minimize the $\mathrm{TE}_{20}$ to $\mathrm{TE}_{10}$ mode coupling, which could otherwise create a monopole and vertical dipole field components in the coupler cell. The CLIC structure with this new coupler concept is shown in Fig. 19. Lossy copper walls have been assumed for simulation. The multipole field components are shown in Fig. 20. Integration of the on-axis electric field gives $-2.52 \times 10^{-5}+1.63 \times 10^{-5} \mathrm{i}$ for $V_{\text {crab }}=1 \mathrm{~V}$ which is of the order of that for the dual-feed structure in Sec. V B. However, there also exists a vertical dipole component excited by the $\mathrm{TE}_{10}$ component as mentioned above. The vertical kick due to this component and horizontal dipole kick are shown in Fig. 21. The integration of vertical kick gives $4.28 \times 10^{-5}-2.5 \times 10^{-3} \mathrm{i} \mathrm{V}$ for a peak horizontal kick of $V_{\text {crab }}=1 \mathrm{~V}$. To overcome the vertical kick the $\mathrm{TE}_{10}$ mode in the $\mathrm{TE}_{20}$ mode guide must be reduced by improving the mode converter. One way of doing this is to 


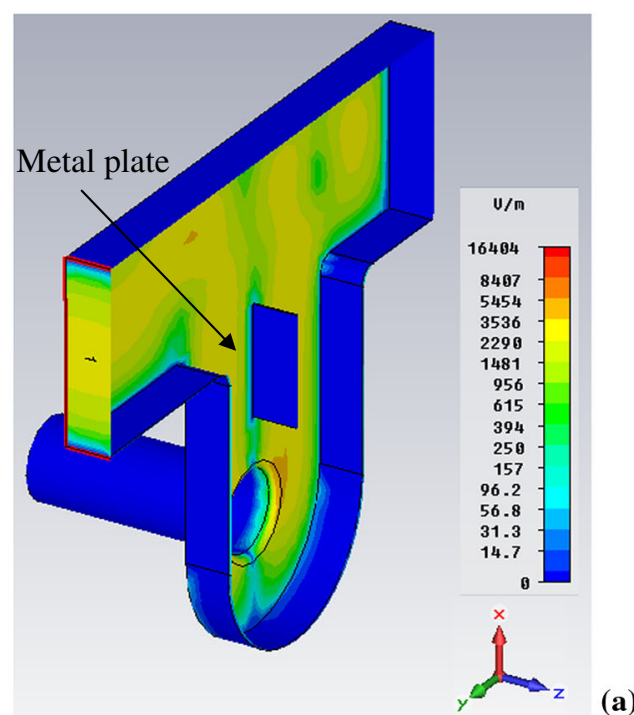

(a)

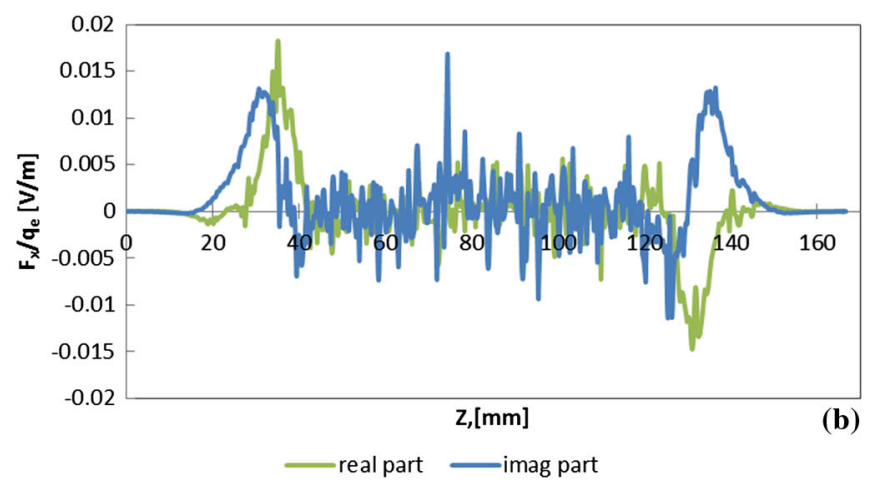

FIG. 22. Vertical kick for the metal plate loaded $\mathrm{TE}_{20}$ coupled cavity for $V_{\text {crab }}=1 \mathrm{~V}$.

electrically short the $\mathrm{TE}_{10}$ field by loading a thin metal plate at $y=$ guide $_{a} / 2$ parallel to the narrow wall of the $\mathrm{TE}_{20}$ guide as shown in Fig. 22(a). The vertical kick is then reduced by about 10 times, comparable to the numerical jitter, as shown in Fig. 22(b). The corresponding vertical kick is $9.93 \times 10^{-6}-2.06 \times 10^{-4} \mathrm{i} \mathrm{V}$ and the monopole voltage is $-2.54 \times 10^{-6}-4.06 \times 10^{-5} \mathrm{i} \mathrm{V}$ for $V_{\text {crab }}=1 \mathrm{~V}$. Table III summarizes the minimum achieved monopole component in all major designs discussed above.

TABLE III. Minimum achieved monopole component for CLIC crab cavity structures discussed. All values correspond to lossy walls and $V_{\text {crab }}=1 \mathrm{~V}$.

\begin{tabular}{lr}
\hline \hline Structure & \multicolumn{1}{c}{ Monopole $\left[\mathrm{V} / \mathrm{m}^{0}\right]$} \\
\hline Single-feed & $-1.05 \times 10^{-3}+1.9 \times 10^{-3} \mathrm{i}$ \\
Dual-feed & $1.32 \times 10^{-5}+3.02 \times 10^{-5} \mathrm{i}$ \\
Single-feed with dummy waveguide & $3.45 \times 10^{-6}-2.32 \times 10^{-4} \mathrm{i}$ \\
Single-feed extra-long end cell & $-1.48 \times 10^{-5}-3.54 \times 10^{-5} \mathrm{i}$ \\
Single-feed-TE ${ }_{20}$ coupler & $-2.52 \times 10^{-5}+1.63 \times 10^{-5} \mathrm{i}$ \\
Single-feed-TE $20^{-}$-coupler- & $-2.54 \times 10^{-6}-4.06 \times 10^{-5} \mathrm{i}$ \\
shorting plate & \\
\hline \hline
\end{tabular}

\section{CONCLUSION}

Various methods to minimize the multipole components of the deflecting mode, especially the monopole field component, present in standard waveguide fed deflecting cavities have been discussed. A detailed study of the causes of monopole field components in deflecting cavities has been performed. The monopole component of the deflecting mode can be caused by asymmetric structures due to the field asymmetries and/or asymmetric power flows. With geometrical modifications, such as a dummy waveguide, the local monopole component can be minimized and by coupler rotation, the total monopole component can be reduced in the steady state. In the case of closely spaced bunches, transient effects of the monopole component can be reduced by using monopole-free coupler designs. A novel $\mathrm{TE}_{20}$ coupler was introduced that is more compact and practical than the conventional dual-feed coupler. The results of this study will lead to simplified monopole-free deflecting structures. This will reduce the footprint of these structures without additional beamloading, which is a major advantage for most applications.

\section{ACKNOWLEDGMENTS}

The research discussed in the paper has been supported by the European Commission under the FP7 Research Infrastructure project EuCARD, Grant Agreement No. 227579 and by STFC through the Cockcroft Institute.

[1] O. A. Altenmueller et al., SLAC Report No. SLAC-R-017, 1963.

[2] R. B. Palmer, SLAC Technical Report No. SLAC-PUB4707, 1988.

[3] T. Abe et al., in Proceedings of the 2007 Particle Accelerator Conference, Albuquerque, New Mexico (IEEE, New York, 2007).

[4] C. Adolphsen et al., EUROTeV-report-2007-010, 2007.

[5] R. Calaga, in the ICFA Beam Dynamics Mini-Workshop on Deflecting/Crabbing Cavity Applications in Accelerators, Cockcroft Institute, 2010 (Cockcroft Institute, Daresbury, Warrington, UK, 2010).

[6] CLIC parameter note, available at http://clic-meeting .web.cern.ch/clic-meeting/.

[7] P. Emma et al., SLAC Report No. SLAC-PUB-8864, 2001.

[8] K. Harkay et al., RF and X-ray Optics Issues in Generating Ultra Short Pulses at APS with Deflecting Cavities, Diamond LS, 2005 (Diamond Light Source, Oxfordshire, UK, 2005).

[9] Z. Li et al., "Cavity BPM with dipole-mode-selective coupler," KEK, SLAC, 2006.

[10] A. Grudiev, S. Calatroni, and W. Wuensch, Phys. Rev. ST Accel. Beams 12, 102001 (2009).

[11] A. Grudiev et al., CLIC Conceptual Design Report No. CERN-ATS-2010-212, 2010. 
[12] C. Nantista et al., KEK Report No. KEK-SLAC ISG, 2002.

[13] C. Nantista, S. Tantawi, and V. Dolgashev, Phys. Rev. ST Accel. Beams 7, 072001 (2004).

[14] S. Doebert et al., in Proceedings of the 8th European Particle Accelerator Conference, Paris, 2002 (EPS-IGA and CERN, Geneva, 2002).

[15] A. Dexter et al., International Workshop on Future Linear Colliders, LCWS 2012, Texas (University of Texas, Arlington, TX, 2012).

[16] A. C. Dexter, G. Burt, P. K. Ambattu, V. Dolgashev, and R. Jones, Nucl. Instrum. Methods Phys. Res., Sect. A 657, 45 (2011).

[17] P. K. Ambattu, G. Burt, V. F. Khan, R. M. Jones, A. Dexter, and V. Dolgashev, Nucl. Instrum. Methods Phys. Res., Sect. A 657, 27 (2011).
[18] N. M. Kroll et al., in Proceedings of the 20th International Linac Conference, Monterey, CA, 2000 (SLAC, Menlo Park, CA, 2000).

[19] J. Barranco Garcia et al., in Proceedings of the 3rd International Particle Accelerator Conference, New Orleans, Louisiana, USA, 2012 (IEEE, Piscataway, NJ, 2012), p. 1873.

[20] W. K. H. Panofsky et al., Rev. Sci. Instrum. 27, 967 (1956).

[21] V. Dolgashev et al., Proceedings of AAC 2012, Texas, 2012 (American Institute of Physics, New York, 2012).

[22] G. Burt et al., in Proceedings of the 3rd International Particle Accelerator Conference, New Orleans, Louisiana, USA, 2012 (Ref. [19]), p. 3335.

[23] V. Sajaev, Beam Dynamics of Chirp Scheme in Storage Rings, Beam Dynamics Mini-Workshop on Deflecting/ Crabbing Cavity, 2012, China. 